\title{
Daytime mechanical ventilation in chronic respiratory insufficiency
}

\author{
B. Schönhofer, M. Geibel, M. Sonneborn, P. Haidl, D. Köhler
}

\begin{abstract}
Daytime mechanical ventilation in chronic respiratory insufficiency. B. Schönhofer, M. Geibel, M. Sonneborn, P. Haidl, D. Köhler. (OERS Journals Ltd 1997.

ABSTRACT: Chronic respiratory insufficiency (CRI) is associated with nocturnal hypoventilation. Treatment with noninvasive mechanical ventilation (NIMV) performed overnight relieves symptoms of hypoventilation and improves daytime blood gases in CRI. In order to test whether the efficacy of NIMV depends on it being applied during sleep, we conducted a prospective case-controlled study comparing daytime mechanical ventilation (dMV) in awake patients with nocturnal mechanical ventilation (nMV) given in equal quantities.

We enrolled 34 clinically stable patients (age 56.1 \pm 12.1 yrs, 20 females, 14 males) with CRI due to restrictive lung and chest wall disorders and neuromuscular disease. Using a prospective case-control design, matched subjects were allocated alternately to $\mathrm{dMV}$ and nMV.

After 1 month of NIMV there was considerable symptomatic improvement in both dMV and nMV patients. There were no significant differences between groups in the improvement in daytime arterial carbon dioxide tension $\left(\mathrm{Pa}_{\mathrm{a}} \mathrm{CO}_{2}\right)(\mathrm{dMV}$ from $7.5 \pm 0.6$ to $5.7 \pm 0.6 \mathrm{kPa}$; $\mathrm{nMV}$ from $7.2 \pm 0.5$ to $5.8 \pm 0.5 \mathrm{kPa}, \mathrm{p}<0.0001$ ) and during the unassisted spontaneous night-time ventilation in terms of transcutaneous $\mathrm{Pa}_{2}, \mathrm{CO}_{2}$ (dMV from $8.4 \pm 1.2$ to $6.6 \pm 0.7 \mathrm{kPa}$; $\mathrm{nMV}$ from $8.2 \pm 1.2$ to $6.8 \pm 0.5 \mathrm{kPa}, \mathrm{p}<0.0001$ ).

We conclude that in many respects, when compared to nocturnal mechanical ventilation, daytime mechanical ventilation in awake patients is equally effective at reversing chronic respiratory insufficiency. Since long-term safety issues were not addressed in this study, we recommend that nocturnal mechanical ventilation should remain the modality of choice for noninvasive mechanical ventilation.

Eur Respir J 1997; 10: 2840-2846.
\end{abstract}

Chronic respiratory insufficiency (CRI) is caused by a relative imbalance between the capacity of the respiratory muscles and the load placed upon them [1]. Noninvasive mechanical ventilation (NIMV) relieves symptoms of hypoventilation and improves daytime blood gases in CRI. The efficacy of nocturnal mechanical ventilation (nMV) has been convincingly demonstrated in patients with CRI due to restrictive lung and chest wall disorders and neuromuscular diseases [2].

Hypoventilation in established CRI is found both during the day while the patient is awake and during sleep in the night. However, compared to daytime, the degree of nocturnal hypoventilation is usually more severe and appears earlier in the clinical course.

Traditionally, mechanical ventilation for CRI is commonly used at night [3-8]. This is done both because $\mathrm{nMV}$ is more convenient to the patients (freeing them for daytime activities), but also because hypoventilation is usually more severe during sleep than during the day [911]. Indeed, some investigators consider that the sleep disordered breathing itself causes CRI [2, 10-12]. Therefore, we performed a prospective case controlled study comparing $\mathrm{nMV}$ and daytime mechanical ventilation (dMV) in patients with CRI in order to investigate whether the therapeutic effect of NIMV necessarily depends on the application being nocturnal.
Krankenhaus Kloster Grafschaft, Zentrum für Pneumologie, Beatmungs- und Schlafmedizin, Schmallenberg - Grafschaft, Germany

Correspondence: B. Schönhofer Krankenhaus Kloster Grafschaft Zentrum für Pneumologie, Beatmungsund Schlafmedizin

57392 Schmallenberg - Grafschaft

Germany

Keywords:

Chronic respiratory insufficiency

daytime mechanical ventilation

nocturnal mechanical ventilation

noninvasive mechanical ventilation

Received: January 23, 1997

Accepted after revision August 31, 1997
Materials and methods

\section{Patients}

The hospital in which this study was carried out is a regional referral centre for patients with CRI. All patients with CRI referred for mechanical ventilation to the hospital within $1 \mathrm{yr}$ were assessed for recruitment to the study. Thus, 34 patients were enrolled. During the study the patients were not on long-term oxygen therapy. The patient's diagnoses and characteristics are presented in table 1 . The protocol was approved by our ethical review committee and all subjects gave their informed written consent to participate.

\section{Measurements}

Baseline measurements were performed prior to the runin period of mechanical ventilation; the normal values for lung function data were those of the European Respiratory Society [13]. We measured spirometry and whole body plethysmography (Masterlab, Jäger, Würzburg, Germany). Peak static inspiratory mouth pressure $(P \mathrm{I}, \mathrm{max})$ was performed using a piezo-electric pressure sensor. $P \mathrm{I}$,max was measured at residual volume at least five times, until a reproducible value was obtained; the maximum value obtained 
Table 1. - Anthropometric, lung function and blood gas data of all patients with daytime mechanical ventilation (dMV) and nocturnal mechanical ventilation (nMV) at baseline

\begin{tabular}{|c|c|c|}
\hline & $\mathrm{nMV}(\mathrm{n}=17)$ & $\mathrm{dMV}(\mathrm{n}=17)$ \\
\hline Post-TBC $\mathrm{n}$ & 7 & 7 \\
\hline Scoliosis $n$ & 6 & 6 \\
\hline Neuromuscular $\mathrm{n}$ & 4 & 4 \\
\hline Sex F/M & $10 / 7$ & $10 / 7$ \\
\hline Age yrs & $55.4 \pm 12.6$ & $56.7 \pm 12.1$ \\
\hline Weight $\mathrm{kg}$ & $61.9 \pm 16.3$ & $61.2 \pm 16.9$ \\
\hline Height $\mathrm{cm}$ & $163.9 \pm 14.1$ & $161.9 \pm 14.8$ \\
\hline $\mathrm{Pa}_{\mathrm{a}, \mathrm{O}_{2}} \mathrm{kPa}^{*}$ & $6.2 \pm 0.8$ & $5.8 \pm 1.0$ \\
\hline 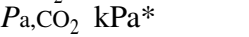 & $7.2 \pm 0.5$ & $7.4 \pm 0.6$ \\
\hline $\mathrm{pH}^{*}$ & $7.35 \pm 0.05$ & $7.34 \pm 0.04$ \\
\hline$P_{0.1 \mathrm{kPa}}$ & $0.4 \pm 0.1$ & $0.4 \pm 0.1$ \\
\hline$P \mathrm{I}, \max \mathrm{kPa}$ & $4.0 \pm 1.5$ & $4.1 \pm 1.5$ \\
\hline$P 0.1 / P \mathrm{I}, \max \%$ & $10.0 \pm 3.2$ & $10.3 \pm 3.6$ \\
\hline$f_{\mathrm{R}}$ & $25.4 \pm 6.0$ & $24.2 \pm 5.0$ \\
\hline$V$ T $\mathrm{mL}$ & $298.0 \pm 81.6$ & $316.0 \pm 91.4$ \\
\hline VC L & $1.1 \pm 0.5$ & $1.1 \pm 0.5$ \\
\hline VC $\%$ pred & $31.8 \pm 6.2$ & $36.4 \pm 8.1$ \\
\hline FEV1 L & $0.7 \pm 0.2$ & $0.8 \pm 0.3$ \\
\hline FEV1 \% pred & $71.9 \pm 13.3$ & $73.3 \pm 7.3$ \\
\hline FEV1 \% 1 VC & $70.7 \pm 14.4$ & $68.4 \pm 19.2$ \\
\hline $\mathrm{Sa}_{\mathrm{a}, \mathrm{O}_{2}}$ mean $\%^{\#}$ & $76.6 \pm 12.9$ & $74.6 \pm 10.6$ \\
\hline $\mathrm{Sa}_{\mathrm{a}, \mathrm{O}_{2}}$ nadir $\%^{\#}$ & $51.5 \pm 12.7$ & $53.3 \pm 12.4$ \\
\hline Mean $P$ tc,, $\mathrm{CO}_{2} \mathrm{kPa}^{\#}$ & $8.4 \pm 1.2$ & $8.6 \pm 1.3$ \\
\hline
\end{tabular}

There were no significant differences between treatments for any variable. TBC: tuberculosis sequelae; $\mathrm{F}$ : female; $\mathrm{M}$; male; $\mathrm{Pa}_{\mathrm{a}} \mathrm{O}_{2}$ : arterial oxygen tension; $P_{\mathrm{a}}, \mathrm{CO}_{2}$ : arterial carbon dioxide tension; $P_{0.1}$ : mouth occlusion pressure; $P \mathrm{I}, \mathrm{max}$ : peak static inspiratory mouth pressure; $f \mathrm{R}$ : breathing rate; $V \mathrm{~T}$ : tidal volume; VC: vital capacity; \% pred: percentage of predicted value; FEV1: forced expiratory volume in one second; $\mathrm{S}_{\mathrm{a}, \mathrm{O}_{2}}$ : arterial oxygen saturation; $P \mathrm{tc}, \mathrm{CO}_{2}$ : transcutaneous carbon dioxide tension. *: during the day; \#: overnight.

was reported [14]. Resting awake respiration was assessed with a portable pneumotachograph (CP100; Bicore, Medilab, Estenfeld, Germany) connected to a mouthpiece yielding tidal volume $(V \mathrm{~T})$ and breathing frequency $(f \mathrm{R})$ while the nose was closed by a clip. Resting daytime capillary blood gases were measured from the hyperaemic earlobe whilst breathing room air. The measurements were repeated after the end of the 1 month period of either dMV or nMV.

The measurements of overnight arterial oxygen saturation $\left(\mathrm{Sa}_{2} \mathrm{O}_{2}\right)$ and transcutaneous partial pressure of $\mathrm{CO}_{2}$ $\left(P \mathrm{tc}, \mathrm{CO}_{2}\right)$ were performed three times. The first occasion was during spontaneous unassisted respiration during sleep, prior to the run-in period. The second was on the first allocated session of mechanical ventilation (whether dMV or nMV) after the run-in period. Additionally capillary blood gases were measured after a $2 \mathrm{~h}$ ventilation period. The third occasion was after the end of the 1 month intervention period, during an overnight period of sleep while breathing spontaneously on room air.

During sleep transcutaneous measurements of $\mathrm{Sa}_{2} \mathrm{O}_{2}$ were made using a pulse oxymeter (Pulsoxy 7; AVL, Bad Homburg, Germany) and a $\mathrm{CO}_{2}$ with transcutaneous capnograph (Tina; Radiometer, Willich, Germany), respectively. The $P$ tc, $\mathrm{CO}_{2}$ infrared sensors, whose temperature was $43^{\circ} \mathrm{C}$, were placed on the upper arm or pectoral region and maintained at $43^{\circ} \mathrm{C}$. In the past, the reliability of the sensor compared to $P \mathrm{a}, \mathrm{CO}_{2}$ was demonstrated [15]. The sensor was calibrated every $6 \mathrm{~h}$ against room air and precision gases (2.5 and 5\% $\mathrm{CO}_{2}$ ). All staff participating in this investigation had extensive in-service education. All signals were transferred from the analogue output via an analogue-todigital converter. After the signals were digitalized, they were processed by computer, analysed and recorded.

\section{Study protocol}

The inclusion criteria were: chronic hypercapnic ventilatory failure (arterial partial pressure of $\mathrm{CO}_{2}\left(\mathrm{~Pa}_{\mathrm{a}} \mathrm{CO}_{2}\right)>5.8$ and $<8.4 \mathrm{kPa}$ ) due to lung or chest wall diseases (posttuberculosis-sequelae and kyphoscoliosis) and neuromuscular diseases despite maximal medical therapy supervised by a chest physician with no hospital admission for at least 1 month prior to the study; and no significant difference between the pre-admission blood gases (1 month preadmission) and those obtained on admission to hospital, indicating that the patients were in a stable state. The upper limit of $\mathrm{Pa}_{\mathrm{a}} \mathrm{CO}_{2}(<8.4 \mathrm{kPa})$ as an inclusion criteria was introduced in order not to expose patients with a very severe degree of CRI to unjustified risk by using an unproven therapy. Subjects were excluded if they had rapidly progressive neuromuscular diseases (e.g. motor neurone disease), obesity-hypoventilation syndrome, chronic obstructive pulmonary disease (COPD), acute respiratory failure (requiring continuous mechanical ventilation), severe acidosis $(\mathrm{pH}<7.3)$, or compromised vision or hearing.

Because CRI can arise from a variety of causes we did not use a simple randomization procedure; instead we used a prospective case control design. Subjects were alternately allocated to dMV and nMV. However, when a subsequent patient entered the study who matched an earlier index subject, he or she was regarded as the control and assigned to the alternative therapy. If he or she did not match an earlier index patient, he or she was regarded as a new index patient. There was no external influence, such as daytime activity, which influenced inclusion into a preferred group. The criteria for a match were appropriate diagnostic group, gender, age, body weight, vital capacity (VC), forced expiratory volume in one second (FEV1), arterial oxygen tension $\left(P \mathrm{a}, \mathrm{O}_{2}\right)$ and $\mathrm{Pa}_{\mathrm{a}} \mathrm{CO}_{2}$. Age, body weight, VC, $\mathrm{FEV} 1, P \mathrm{a}, \mathrm{O}_{2}$ and $\mathrm{Pa}, \mathrm{CO}_{2}$ had to be within $10 \%$ of the corresponding index patient. The end points of the study were daytime blood gases, $f \mathrm{R}, V \mathrm{~T}, P \mathrm{I}$,max, nocturnal $\mathrm{Sa}, \mathrm{O}_{2}$ and $P \mathrm{tc}, \mathrm{CO}_{2}$ during spontaneous breathing.

All patients had a run-in period of $\mathrm{dMV}$ of 5 days to determine the optimal ventilator settings. During this time they were instructed in the use of the interface fit. All patients were initially ventilated via a conventional nose mask (Respironics, Murrysville, USA and Res-care, Sydney, Australia). If pressure sores developed or if the quality of ventilation worsened due to leakage from the mask or the mouth, an individual nose or nose-mouth mask was made by a dental laboratory.

During wakefulness, patients had to fulfil three conditions in order to be judged as having been adequately treated with mechanical ventilation:

1) The absence of spontaneous breathing activity, assessed by an experienced therapist observing the interaction between patient and ventilator. The patient was considered to be passively ventilated if within a $30 \mathrm{~min}$ period fewer than 5\% triggered breaths were registered.

2) Mild hypocapnia $\left(\mathrm{Pa}_{\mathrm{a}} \mathrm{CO}_{2} 4.7-5.3 \mathrm{kPa}\right)$ and alkalosis (pH 7.40-7.45), respectively, during NIMV.

3) No significant mask or oral air leak. 
Table 2. - Ventilator settings during the adaptation period of interventional ventilation in the dMV and nMV group

\begin{tabular}{lcc}
\hline & $\mathrm{dMV}$ & $\mathrm{nMV}$ \\
\hline Tidal volume $\mathrm{mL}$ & $565.3 \pm 123.1$ & $523.3 \pm 91.3$ \\
Inspiration time s & $1.4 \pm 0.2$ & $1.3 \pm 0.3$ \\
Ventilation rate breaths $\cdot \mathrm{min}^{-1}$ & $20.9 \pm 3.3$ & $21.2 \pm 3.9$ \\
$\mathrm{Sa}_{\mathrm{a}} \mathrm{O}_{2}$ mean \% & $94.1 \pm 2.4$ & $93.1 \pm 2.8$ \\
$\mathrm{Sa}_{2}$ nadir \% & $92.1 \pm 1.9$ & $91.8 \pm 2.0$ \\
$\mathrm{Mean} P \mathrm{Pc}, \mathrm{CO}_{2} \mathrm{kPa}$ & $5.1 \pm 0.6$ & $5.4 \pm 0.5$ \\
$\mathrm{~Pa}_{2} \mathrm{O} \mathrm{kPa}$ & $9.8 \pm 0.9$ & $10.0 \pm 1.0$ \\
$\mathrm{~Pa}_{2} \mathrm{CO}_{2} \mathrm{kPa}$ & $4.6 \pm 0.6$ & $4.6 \pm 0.4$ \\
$\mathrm{pH}$ & $7.43 \pm 0.04$ & $7.41 \pm 0.04$ \\
$\mathrm{HCO}_{3}{ }^{-} \mathrm{mmol} \cdot \mathrm{L}^{-1}$ & $23.4 \pm 2.2$ & $22.5 \pm 2.3$ \\
\hline
\end{tabular}

There were no significant differences between treatments for any variable. For further definitions see legend to table $1 . \mathrm{HCO}_{3}^{-}$: bicarbonate.

The groups did not differ significantly with respect to the setting of the ventilator (table 2). Moreover, as judged by $\mathrm{Sa}, \mathrm{O}_{2}$ and $P \mathrm{tc}, \mathrm{CO}_{2}$ during the adaptation period of interventional ventilation, both groups received ventilatory support of equal efficacy. The $P \mathrm{a}, \mathrm{CO}_{2}$ reduction during mechanical ventilation was associated with a mild alkalosis.

We used a volume cycled technique (either Dräger EV 800 (Lübeck, Germany) or PLV 100, (Lifecare, Denver, CO, USA)). In accordance with the study protocol, the patients were ventilated with volume-cycled ventilators in the controlled mode without receiving oxygen. Before NIMV was started, $f \mathrm{R}$ of the spontaneously breathing patient was determined using a portable pneumotachograph, while the patient was in a sitting position without receiving supplemental oxygen. Intermittent positive pressure ventilation (IPPV) was then started, with a pneumotachograph inserted in the tubing system between mask and expiratory valve of the ventilator. The rate of the unit was set at the same rate or slightly higher than that measured during spontaneous breathing in order to suppress the respiratory activity of the patient. The inspiration time was chosen, according to the underlying disease and the patients' subjective well-being, between 40 and $50 \%$. The tidal volume was chosen between $8-12 \mathrm{~mL} \cdot \mathrm{kg}^{-1}$ body weight.

The settings thus determined were not altered, irrespective of which group the patients were subsequently allocated to. After the run-in period, patients were allocated either to nMV (continuously for $8 \mathrm{~h}$ ) or dMV, which was performed in four sections with each part lasting $2 \mathrm{~h}$ (06:00-08:00 h, 11:00-13:00 h, 16:00-18:00 h, 21:0023:00 h). At the end of the 1 month period, all subjects were studied overnight during spontaneous unassisted breathing measuring $\mathrm{Sa}_{\mathrm{a}} \mathrm{O}_{2}$ and $P \mathrm{tc}, \mathrm{CO}_{2}$. Both the dMV and $\mathrm{nMV}$ group stayed in the high dependency unit of our hospital for the duration of the study to ensure that patients performed NIMV correctly.

For the nMV group, sleep was not quantified, although all subjects considered they had an adequate night's sleep. Furthermore, the nursing staff of the high dependency ward registered whether the patients slept by documenting the hourly observations from 22:00 to 06:00 h.

For the dMV group, strict measures were used to ensure wakefulness during dMV. Intensive interaction with the staff and relatives was encouraged by, for example, handicrafts and solving crossword puzzles. In addition, an alertgenerator was constructed, which generated optical signals at random intervals at an average rate of 15 signals $\cdot \mathrm{h}^{-1}$ and which had to be turned off within $30 \mathrm{~s}$. If the optical signal was missed an acoustic signal had to be turned off within $10 \mathrm{~s}$. If both signals were missed, the caring nurse was alarmed. Patients who missed more than $5 \%$ of the signals were removed from the study.

\section{Statistical analysis}

Results are expressed as mean \pm SD. Wilcoxon test was used for the intra-individual comparison of the patients within each group. The significance for two samples was determined by the Mann-Whitney U-test (two-sided). In all cases, a p-value of less than 0.05 was considered significant.

\section{Results}

Anthropometic and diagnostic data are presented in table 1. All patients had CRI with daytime hypercapnia and hypoxaemia; $f \mathrm{R}$ was increased and $\mathrm{pH}, P \mathrm{I}, \max , V \mathrm{~T}$, $\mathrm{VC}, \mathrm{FEV} 1$ and $\mathrm{Sa}_{\mathrm{a}} \mathrm{O}_{2}$ were reduced (table 1) in comparison with published normal values [13, 14, 16, 17]. In particular, all patients had evidence of nocturnal hypoventilation characterized by an increase of nocturnal hypercapnia compared to the daytime values.

Table 3. - Physiological parameters at baseline and after 1 month of daytime mechanical ventilation (dMV) and 1 month nocturnal mechanical ventilation (nMV) during spontaneous breathing

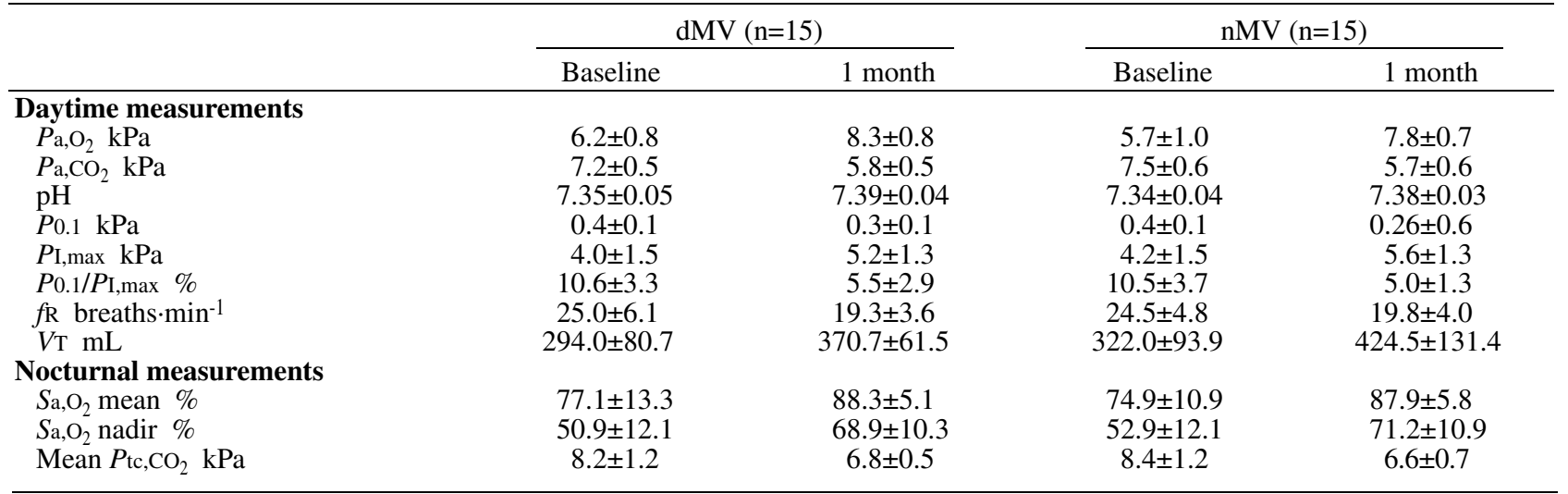

For both nMV and dMV, the baseline and 1 month values of all parameters were significantly different $(\mathrm{p}<0.001)$. For definitions see legend to table 1. 
a)
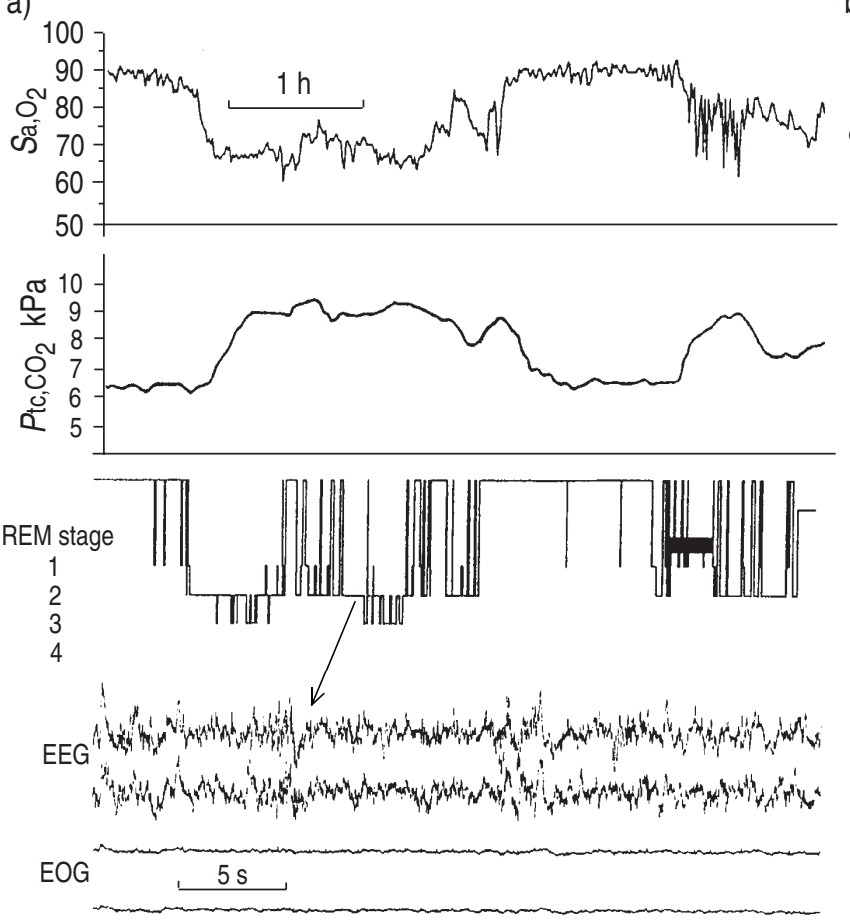

EMG

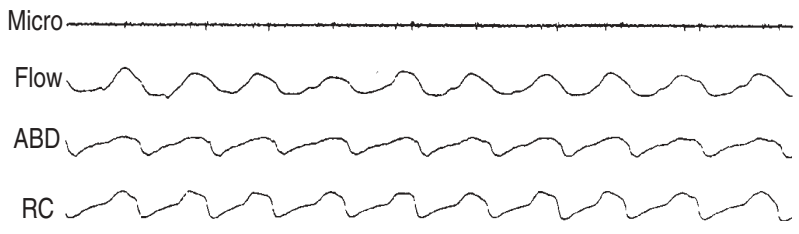

b)
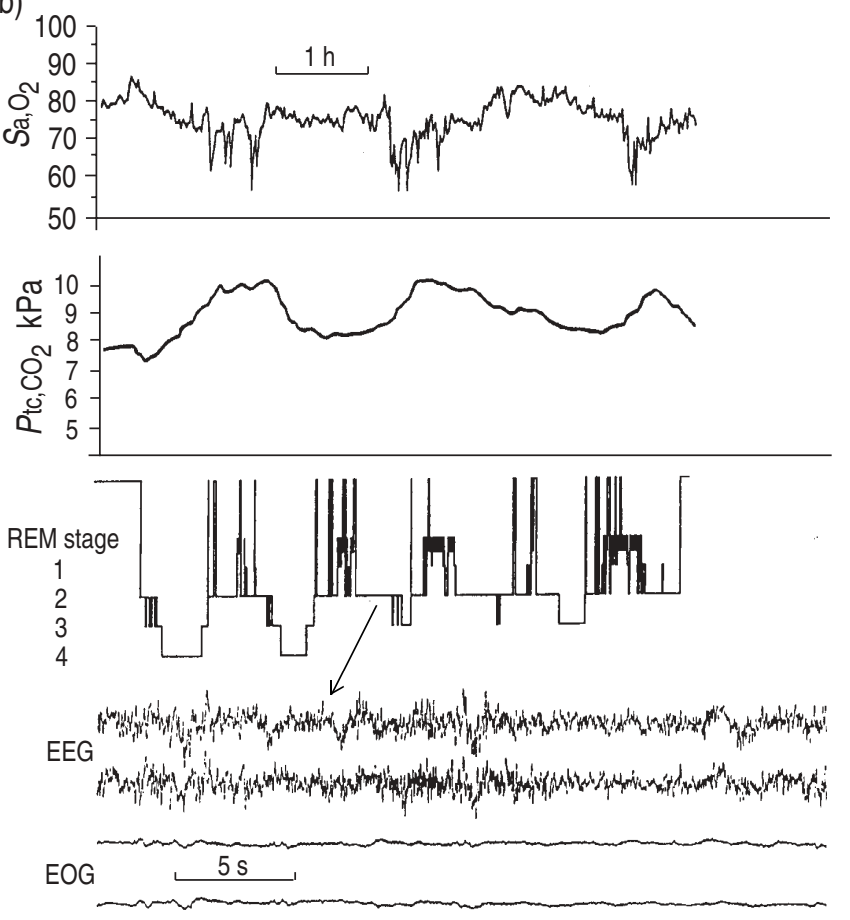

EMG

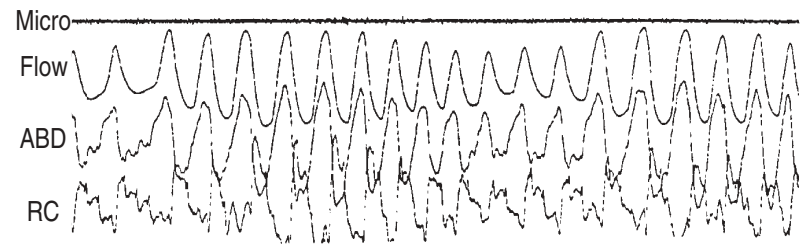

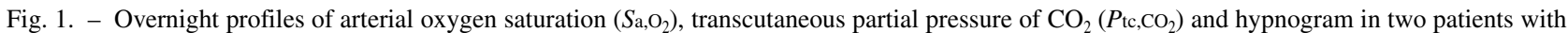
chronic respiratory insufficiency due to kypohoscoliosis. Measurements were performed during unassisted ventilation while breathing room air before commencing either: a) nocturnal mechanical ventilation (female $40 \mathrm{yrs}$ ); or b) daytime mechanical ventilation (female, 38 yrs). The channels of the polysomnographic tracing in this $30 \mathrm{~s}$ period of nonrapid eye movement (NREM) show, from top to the bottom: electroencephalogram (EEG), electrooculogram (EOG), submental and tibial electromyogram (EMG), microphone (micro), oronasal flow and excursions of the abdomen (ABD) and rib cage (RC) Persisting nocturnal hypoventilation with low arterial oxygen saturation $\left(\mathrm{Sa}_{\mathrm{a}} \mathrm{O}_{2}\right)$ and hypercapnia occurred continuously throughout the night, further increasing in rapid eye movement (REM) sleep. The pattern of breathing in NREM sleep was rapid and shallow.

Since we used a prospective case control design it is not unexpected that at baseline neither groups differed with respect to the matching criteria (age, body weight, $\mathrm{VC}$, $\left.\mathrm{FEV} 1, P \mathrm{a}, \mathrm{O}_{2}, P \mathrm{a}, \mathrm{CO}_{2}\right)$. Furthermore, there were no significant differences between the dMV and the nMV groups in $f \mathrm{R}, V \mathrm{~T}$, mouth occlusion pressure $\left(P_{0.1}\right), P_{\mathrm{I}}$,max and the measured parameters during nocturnal ventilation $\left(\mathrm{Sa}_{\mathrm{a}} \mathrm{O}_{2}\right.$, $P \mathrm{tc}, \mathrm{CO}_{2}$ ) at baseline (table 1 ).

In the dMV group more than $90 \%$ of the optical alarms were turned off by the patients, confirming that they were awake. Two of 17 patients were not able to stay awake during dMV and were, therefore, excluded from further analysis, as were the corresponding patients allocated to nMV, leaving a total of 30 subjects. According to the visual observations all nMV patients slept for at least $80 \%$ of the observed period from 22:00 to 06:00 $\mathrm{h}$.

All patients experienced symptomatic relief following the institution of mechanical ventilation. After 1 month of both $\mathrm{dMV}$ and $\mathrm{nMV}$, the following parameters measured during spontaneous breathing showed significant improvement in both groups (table 3): $P \mathrm{a}, \mathrm{CO}_{2}$ and $\mathrm{RR}$ decreased; and $P \mathrm{a}, \mathrm{O}_{2}, P \mathrm{I}$,max, $V \mathrm{~T}$, and $\mathrm{pH}$ increased (all $\mathrm{p}<0.0001$ ), confirming efficacy of the treatment. There was no difference in the magnitude of improvement according to whether the patient had been allocated to dMV or nMV.

Comparing spontaneous breathing during sleep, after 1 month NIMV both groups showed an improvement of ventilation, increasing both the mean and nadir $\mathrm{Sa}_{\mathrm{a}} \mathrm{O}_{2}$ ( $\mathrm{p}<0.0001)$; correspondingly the mean $\mathrm{Ptc}_{\mathrm{tc}} \mathrm{CO}_{2}$ decreased (table 3). However, nocturnal hypoventilation only partially normalized, since, when compared to values obtained during nMV, the levels of $\mathrm{Sa}_{\mathrm{a}} \mathrm{O}_{2}$ and $P \mathrm{tc}, \mathrm{CO}_{2}$ were worse during nocturnal unassisted spontaneous breathing in both groups, even after 1 month of treatment (tables 2 and 3). Although not part of the study design, a sleep study was preformed in some patients of each group. Figures 1 and 2 illustrate typical polysomnographic examples of both the $\mathrm{nMV}$ and dMV group in the course of the study period. 

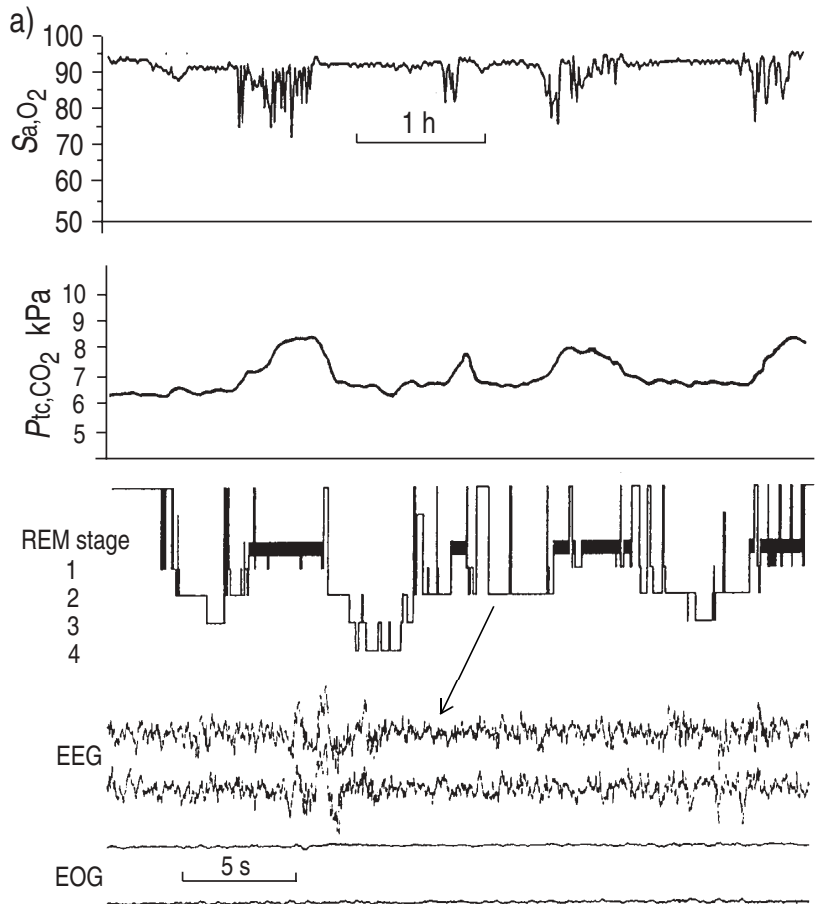

EMG

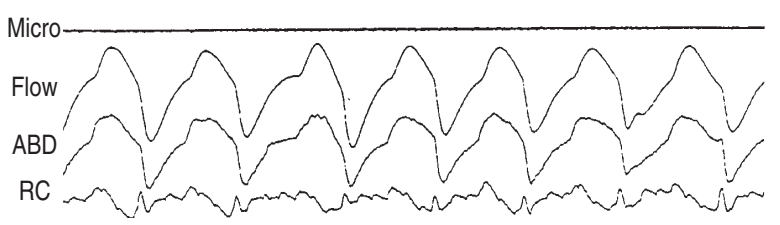

b)
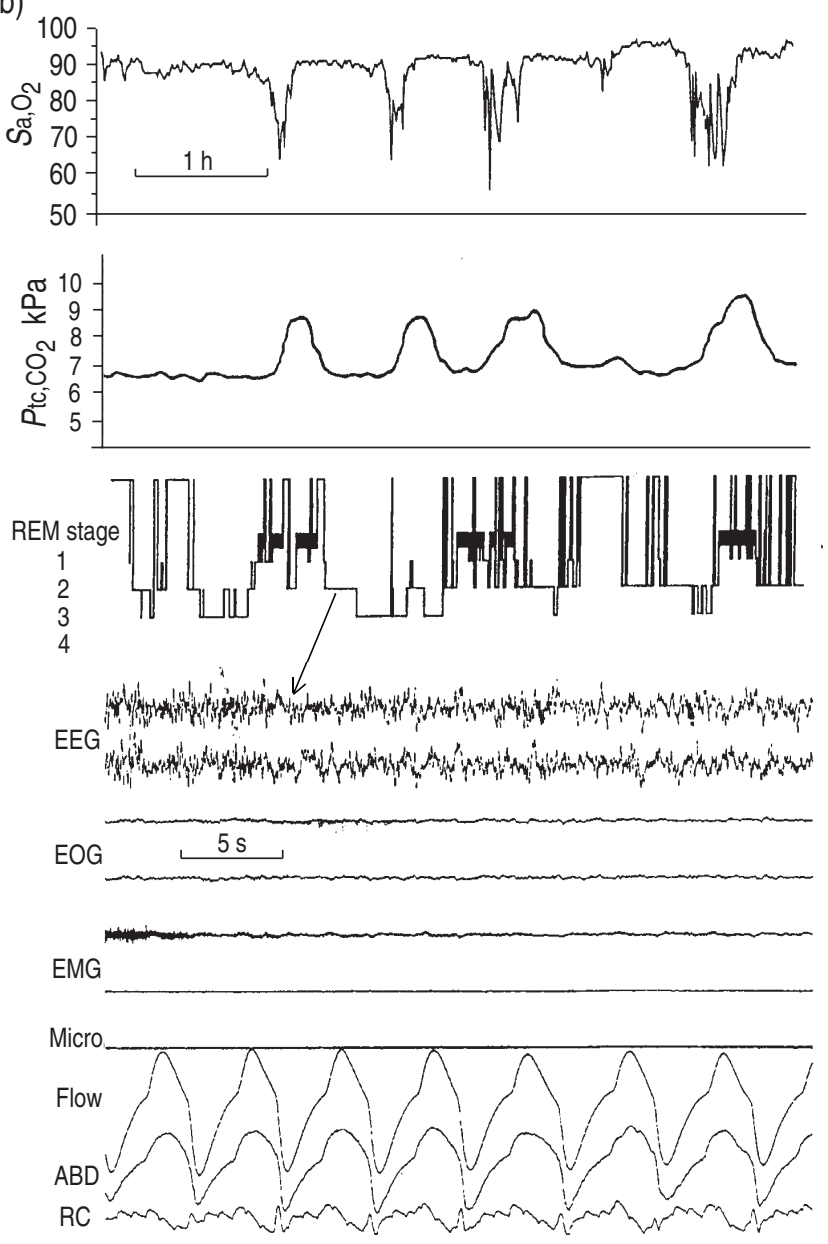

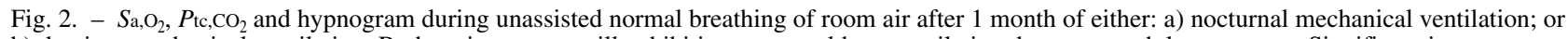
b) daytime mechanical ventilation. Both patients were still exhibiting nocturnal hypoventilation, but to a much lesser extent. Significant improvement of ventilation $\left(\mathrm{Sa}_{\mathrm{a}, \mathrm{O}_{2}}\right.$ and $\left.P \mathrm{tc}, \mathrm{CO}_{2}\right)$ during NREM-sleep and episodic nonapnoeic hypoventilation during REM-sleep. Accordingly, RC and ABD changed during spontaneous breathing in NREM sleep: the breathing frequency decreased compared to before noninvasive mechanical ventilation (fig. 1). Furthermore, after the intervention both patients showed an improvement of sleep architecture (increased REM and slow wave sleep). For definitions see legend to figure 1.

The effort traces show a substantial improvement of the unassisted nocturnal ventilation and sleep architecture after 1 month NIMV, irrespective of group (for further explanation see legend to figures 1 and 2).

\section{Discussion}

This study showed that the efficacy of NIMV does not depend on the application during sleep. Patients with CRI are equally well and effectively ventilated during both the night and the day. The effect of NIMV is not determined by whether the patients are awake or asleep when receiving it. Before discussing this finding further, criticisms of the methods will be addressed.

Although nocturnal hypoventilation was not a matching criteria, in fact the dMV and nMV group showed no significant difference with respect to $\mathrm{CO}_{2}$ retention and hypoxaemia in the entry sleep study, indicating a comparable degree of hypoventilation during the night. Although $\mathrm{nMV}$ and dMV were equally effective with respect to daytime arterial blood gases, the situation at night as judged by nocturnal $\mathrm{Sa}, \mathrm{O}_{2}$ and $P \mathrm{tc}, \mathrm{CO}_{2}$ was not optimal for the dMV group since these parameters did not completely normalize during spontaneous nocturnal breathing after 1 month (see table 3), whereas the nMV group received mechanical ventilation during each night. Thus, at least for a time, remaining nocturnal hypoventilation during spontaneous breathing (which is probably associated with REM sleep) could have undesirable cardiovascular or haematologist consequences in the long term since the pulmonary artery pressure is supposed to be increased during this period. Our data, therefore, do not support long-term use of $\mathrm{dMV}$, except where $\mathrm{nMV}$ is not tolerated by the patients.

Recently, it has been shown that the mode of mechanical ventilation may induce reduction of effective ventilation by narrowing the glottis $[18,19]$. Thus, although the ventilator settings were not influenced by allocation to the $\mathrm{dMV}$ or $\mathrm{nMV}$ group, it is possible that the $\mathrm{dMV}$ group were effectively receiving more ventilation than the nMV group. We acknowledge that without systematic measurement of ventilation (rib cage and abdominal movement) 
we are not able to exclude this phenomenon for the present data. However, this does not detract from our main observation that NIMV need not be given during sleep in order to be effective.

We acknowledge that the lack of sleep studies weakens the conclusions, but the overnight $S \mathrm{a}, \mathrm{O}_{2}$ and $P \mathrm{tc}, \mathrm{CO}_{2}$ were measured during spontaneous breathing both at baseline and following the intervention. Since the study population exclusively consisted of patients with restrictive pulmonary diseases, the documented nocturnal decrease in $\mathrm{Sa}_{\mathrm{a}} \mathrm{O}_{2}$ desaturation and the increase in $P \mathrm{tc}, \mathrm{CO}_{2}$ are sufficient evidence of hypoventilation during sleep. Although the degree of sleep disordered breathing was not quantified using polysomnography in the nMV group, it seems reasonable to assume that they were asleep, since they were monitored by the nursing staff who confirmed the patients being asleep for more than $80 \%$ of the observed periods. Furthermore, we cannot absolutely exclude the possibility that short periods of sleep could have occurred during mechanical ventilation in the dMV group. However, the alert generator excluded overt periods of sleep during dMV.

The ideal way to compare two interventions is to perform a prospective controlled randomized study. However such a design would require the enrolment of a large number of patients to ensure equal distribution for the nMV and dMV groups; such a study would probably not be realistic for a single institution. Therefore, we opted for a prospective case control design. Our data show that the nMV and dMV groups were closely comparable with respect to physiological indices of CRI severity. Therefore, we are also reasonably confident that biases in exclusion of patients did not alter our conclusions.

The observation that $\mathrm{dMV}$ is an effective therapy for CRI is of great physiological interest. Treatment, using $\mathrm{nMV}$, of sleep-disordered breathing due to CRI is an established therapy, and has been shown to result in normalization of nocturnal blood gases and reversal of daytime respiratory failure $[2,12,20]$. In the present study we have shown that dMV leads to an improvement in the measured parameters during the daytime without a direct treatment of the associated sleep disordered breathing itself. This implies that improvement obtained with $\mathrm{dMV}$ is not directly mediated by an effect on sleep quality. Furthermore, since our dMV protocol used four discrete $2 \mathrm{~h}$ intervals, it does not seem necessary to have $8 \mathrm{~h}$ of continuous mechanical ventilation to provide effective therapy. In this context it is generally agreed that spontaneous ventilation is worst during sleep in CRI [9-11, 21]; this results in part from lung mechanics in the supine position [22, 23], pathological breathing pattern [22] and reduced chemosensitivity during rapid eye movement (REM) sleep [9]. Our data, therefore, suggest that mechanical ventilation does not need to be directed at what is, functionally, the worst period of hypoventilation. CRI may, at least partially, be reversed without primarily preventing sleep disordered breathing. Thus, sleep disordered breathing is shown to be an associated phenomenon rather than inducing CRI itself.

Our finding that similar benefit is obtained from dMV and nMV neither exclude nor favour one of the two main hypotheses that NIMV leads to respiratory muscle rest and/or resetting the chemosensitivity $[2,11,24]$. If muscle rest were the main mechanism, NIMV should reduce respiratory muscle activity leading to improvement of muscle strength and endurance [24]. In our study, maximal inspir- atory muscle strength improved in all patients. Previously reported data regarding the effect of NIMV on PI,max values are conflicting, muscle strength has been found to be either unchanged $[6,25,26]$ or improved $[5,10,12]$. The principal problem is that the PI,max test is volitional and increase in strength may be related to motivational factors or a learning effect rather than an im-provement in muscle function per se.

However, according to the resetting hypothesis, NIMV works by counteracting the reduction in central drive associated with hypoventilation and the consecutive rise in $P \mathrm{a}, \mathrm{CO}_{2}[2,12,24]$. In this model, renal retention of bicarbonate is increased in order to maintain $\mathrm{pH}$ near normal. Consequently, the central $\mathrm{CO}_{2}$ receptors are thought to be blunted and ventilatory failure ensues. If chemosensitivity is restored by applying NIMV, whether by day or night, excretion of bicarbonate and central drive should increase and ventilation should improve inherently during spontaneous breathing. We demonstrated in both groups that NIMV reduces $\mathrm{CO}_{2}$ and leads to a mild alkalosis.

Thus, our data could be used to support both hypotheses. In order to study these hypotheses definitively it would be desirable to examine both the changes in respiratory drive in response to hypercapnia and the change in muscle strength, for example by using the nonvolitional phrenic nerve stimulating technique [27]. We acknowledge that these data are not provided by the present study and, thus, the mechanism of improvement remains speculative. To some extent this is inherent in studies using the model of mechanical ventilation, since if the treatment is performed effectively, the hypercapnia is prevented and, simultaneously, the respiratory muscles are at least partly rested. However, the concept of dMV described here could prove a useful paradigm for future studies in this area.

Anecdotally, dMV has been described in the past [28]. We do not suggest that dMV is superior to nMV, even though we demonstrated its efficacy. Indeed the advantage that nMV leaves the patient free to pursue the activities of daily living is considerable. All but two patients changed from dMV to nMV after the study finished. The two patients who chose to remain with dMV did so because of the anxiety and handling problems with the nose mask during sleep. However dMV may occasionally be indicated for those patients who cannot retain the mask or mouthpiece due to oral weakness.

We conclude that in awake and clinically stable patients, compared to nocturnal mechanical ventilation, daytime mechanical ventilation reverses chronic respiratory insufficiency to equal quantities over a 1 month period. Therefore, our data question the assumption that sleep disordered breathing itself is a mandatory cause of chronic respiratory insufficiency. It rather seems to be an associated phenomenon. Long-term safety issues (such as cardiovascular or haemodynamic adverse effects due to the remaining component of nocturnal hypoventilation) were not addressed in this study; therefore, we recommend that nocturnal mechanical ventilation should remain the modality of choice for noninvasive mechanical ventilation.

Acknowledgements: The authors thank $M$. Polkey and M. Elliott for their critical remarks and linguistic advice on the article. They are grateful to M. Klauke for technical assistance and for constructing the alert generator in particular. 


\section{References}

1. Goldstone J, Moxham J. Weaning from mechanical ventilation. Thorax 1991; 46: 56-62.

2. Meyer TJ, Hill NS. Noninvasive positive pressure ventilation to treat respiratory failure. Ann Intern Med 1994; 120: 760-770.

3. Bach JR, Alba AS, Saporito LR. Intermittent positive pressure ventilation via the mouth as an alternative to tracheostomy for 257 ventilator users. Chest 1993; 103: 174-182.

4. Carroll N, Branthwaite MA. Control of nocturnal hypoventilation by nasal intermittent positive pressure ventilation. Thorax 1988; 43: 349-353.

5. Ellis RE, Bye PT, Bruderer JW, Sullivan CE. Treatment of respiratory failure during sleep in patients with neuromuscular disease. Positive-pressure ventilation through a nose mask. Am Rev Respir Dis 1987; 135: 148-152.

6. Gay PC, Patel AM, Viggiano RW, Hubmayr RD. Nocturnal nasal ventilation for treatment of patients with hypercapneic respiratory failure. Mayo Clinic Proc 1991; 66: 695-703.

7. Leger P, Bedicam JM, Cornette A, et al. Nasal intermittent positive pressure ventilation. Chest 1994; 105: 100-105.

8. Simonds AK, Elliott MW. Outcome of domiciliary nasal intermittent positive pressure ventilation in restrictive and obstructive disorders. Thorax 1995; 50: 604-609.

9. Goldstein RS, Molotiu N, Sktrastins R, et al. Reversal of sleep-induced hypoventilation and chronic respiratory failure by nocturnal negative pressure ventilation in patients with restrictive ventilatory impairment. Am Rev Respir Dis 1987; 135: 1049-1055.

10. Jimenez JFM, Sanchez de Cos Escuin J, Vicente CD, Valle MH, Otero FF. Nasal intermittent positive pressure ventilation. Chest 1995; 107: 382-388.

11. Hill NS. Noninvasive ventilation. Am Rev Respir Dis 1993; 147: 1050-1055.

12. Piper AJ, Sullivan CE. Effects of long-term nocturnal nasal ventilation on spontaneous breathing during sleep in neuromuscular and chest wall disorders. Eur Respir $J$ 1996; 9: 1515-1522.

13. Quanjer PH, Dalhuijsen A, Van Zomeren BC. Summary equations of reference values. Bull Eur Physiopathol Respir 1983; 19 (Suppl. 5): 45-51.
14. Black LF, Hyatt RE. Maximal respiratory pressures: normal values and relationship to age and sex. Am Rev Respir Dis 1969; 99: 696-702.

15. Brambilla I, Micallef E, Sacerdoti C, Arlati S, Rolo J. Value of nocturnal monitoring of transcutaneous $\mathrm{O}_{2}$ and $\mathrm{CO}_{2}$ pressures in adults with respiratory failure. Respiration 1985; 48: 81-90.

16. American Thoracic Society. Lung function testing: selection of reference values and interpretative strategies. $A m$ Rev Respir Dis 1991; 144: 1202-1218.

17. Cerveri I, Zoia MC, Fanfulla F, et al. Reference values of arterial oxygen tension in the middle-aged and elderly. Am J Respir Crit Care Med 1995; 152: 934-941.

18. Jounieaux V, Aubert G, Dury M, Delguste P, Rodenstein DO. Effects of nasal positive pressure hyperventilation on the glottis in normal awake subjects. J Appl Physiol 1995; 79: 176-185.

19. Parreira VF, Jounieaux V, Aubert G, Dury M, Delguste PE, Rodenstein DO. Nasal two level positive-pressure ventilation in normal subjects. Am J Respir Crit Care Med 1996; 153: 1616-1623.

20. Claman DM, Piper A, Sanders MH, Stiller RA, Votteri BA. Nocturnal noninvasive positive pressure ventilatory assistance. Chest 1996; 110: 1581-1588.

21. Piper AJ, Sullivan CE. Sleep-disordered breathing in neuromuscular disease. In: Saunders NA, Sullivan CE, eds. Sleep and breathing. 2nd ed. 1994; pp. 760-786.

22. Bergofsky EH. Respiratory failure in disorders of the thoracic cage. Am Rev Respir Dis 1979; 119: 643-669.

23. Newsom DJ, Loh LM, Casson M. Diaphragm function and alveolar hypoventilation. $Q$ J Med 1976; 45: 87-100.

24. Martin TJ, Sanders MH. Chronic alveolar hypoventilation: a review for the clinician. Sleep 1995; 18: 617-634.

25. Goldstein RS, Rosie JA, Avendano MA, Dolmage TE. Influence of noninvasive pressure ventilation on inspiratory muscles. Chest 1991; 99: 408-415.

26. Kerby GR, Mayer LS, Pingleton SK. Nocturnal positive pressure ventilation via nasal mask. Am Rev Respir Dis 1987; 135: 738-780.

27. Polkey MI, Green M, Moxham J. Measurement of respiratory muscle strength. Thorax 1995; 50: 1131-1135.

28. Bach JR. Update and perspectives on noninvasive respiratory muscle aids. Chest 1994; 105: 1230-1240. 